\title{
Irreversible Otto heat engine with friction and heat leak losses and its parametric optimum criteria
}

\author{
Y. R. Zhao and J. C. Chen*
}

\begin{abstract}
A more realistic simplified model for an irreversible Otto cycle is established by considering the heat leak losses through the cylinder and internally dissipative friction, in which it is assumed that the piston velocity in the adiabatic processes obeys a pure sinusoidal law. The optimally operating region of the irreversible Otto heat engine is determined. The model avoids the usual hypotheses of endoreversible heat engines and the simple description of irreversible heat engines so that the results obtained here may include the performance characteristics of some simplified Otto cycle models.
\end{abstract}

Keywords: Otto heat engine, Friction, Heat leak, Maximum work and efficiency, Optimum criterion

\section{Introduction}

A study on gas cycles as models of internal combustion engines is useful for illustrating some of the important parameters influencing engine performance. Many significant achievements have been made to analyse and optimise the performance of the Otto heat engine. ${ }^{1-9}$ An irreversible simplified model for the air standard Otto cycle was proposed to study the effect of friction losses on the performance of the cycle. ${ }^{2-4}$ The analyses can be drawn from two main hypotheses:

(i) the instantaneous character of the adiabatic strokes, as usual in endoreversible heat engines

(ii) the gathering of the global losses in a dissipation term represented by a friction force linear in the piston mean velocity.

These hypotheses present unrealistic results and make the Otto cycle unfeasible to a certain extent.

The aim of this paper is to present an alternative analysis of an irreversible Otto cycle. Based on the work by Hernández et al. ${ }^{10}$ the authors consider the explicit piston velocity depending on its displacement in the adiabatic processes. The proposed model results in qualitative and quantitative agreement with real heat engine results for the work output, thermal efficiency and work versus efficiency behaviour.

\section{Irreversible cycle model}

An Otto cycle is composed of two adiabatic and two isochoric processes, as shown in Fig. 1, where the adiabatic compression and expansion processes 1-2S and 3-4S are reversible, while $1-2$ and $3-4$ are irreversible. The volumes of working substance in two isochoric processes are presented by $V_{1}$ and $V_{2}$, and the

Department of Physics, Xiamen University, Xiamen 361005, China

*Corresponding author, email jcchen@xmu.edu.cn temperatures of working substance in state points $1,2 \mathrm{~S}$, 2, 3, 4 and $4 \mathrm{~S}$ are presented by $T_{1}, T_{2 \mathrm{~S}}, T_{2}, T_{3}, T_{4}$ and $T_{4 \mathrm{~S}}$ respectively. $T_{1}$ and $T_{3}$ are the lowest and highest temperatures of working substance in the cycle respectively, which are determined by the ambient air conditions and combustion characteristics of the gasoline-air mixture, and may be taken as two constants. ${ }^{11}$ Here, the authors consider an irreversible Otto cycle consisting of states 1-2-3-4-1.

In practice, heat leak and friction losses reduce the efficiency of real heat engine by about $40 \%{ }^{1}$ Friction typically dissipates about $20 \%$ of the power developed by the engine. Of this about $75 \%$ is due to the friction of piston rings on the cylinder walls. For this contribution one assumes a friction force linear in velocity, ${ }^{1}$ i.e.

$$
F_{\mu}=\mu v
$$

where $\mu$ is the friction coefficient, and $v$ is the velocity of piston. Due to greater pressure on the piston after combustion, the value of $\mu$ is usually about twice as large on the power stroke as on the compression stroke. ${ }^{1}$ It means that $\mu_{\mathrm{P}}=2 \mu_{\mathrm{C}}$, where $\mu_{\mathrm{C}}$ and $\mu_{\mathrm{P}}$ are friction coefficients on the compression and power strokes respectively.

As described by Hernández et al. ${ }^{10}$ one may make the following hypotheses:

(i) irreversibility in the adiabatic processes is only due to internally dissipative friction with frictional losses being directly dissipated to the surroundings

(ii) on the adiabatic strokes, the piston velocity $v(x)$ follows a pure sinusoidal law with the piston displacement $x$, which takes zero value in their extreme points. This modelisation of the piston motion is valid when no constraints on the acceleration are taken into account ${ }^{1}$ and it is also compatible with the isochoric character given to the heating and cooling processes. 


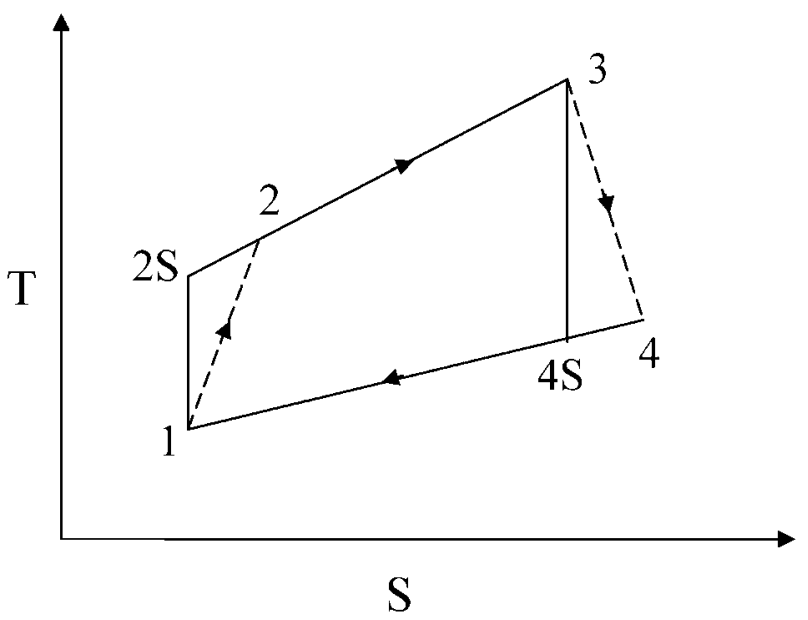

1 Schematic diagram of irreversible Otto heat engine

According to hypothesis (ii), one may take the piston velocity in adiabatic processes as

$$
v(x)=v_{\max } \sin (k x+\phi)
$$

where $v_{\max }, k$ and $\phi$ are characteristic parameters of the piston motion. Given null value of the velocity at the extreme points $X_{1}$ and $X_{2}$, it can be obtained from equation (2) that $k X_{1}+\phi=0$ and $k X_{2}+\phi=\pi$, so that one has $k\left(X_{2}-X_{1}\right)=\pi$, i.e.

$$
k=\frac{\pi}{X_{2}-X_{1}}=\frac{\pi}{X_{2}\left(1-r_{\mathrm{v}}\right)}<0
$$

with $r_{\mathrm{v}}=X_{1} / X_{2}=V_{1} / V_{2} \geqslant 1$ being the compression ratio of the cycle. From equations (1)-(3), one can calculate the friction work as

$$
\left\{\begin{array}{l}
W_{\mu_{\mathrm{C}}}=-\int_{\mathrm{X}_{1}}^{\mathrm{X}_{2}} F_{\mu_{\mathrm{C}}}(x) \mathrm{d} x=\frac{2 v_{\max } \mu_{\mathrm{C}} X_{2}}{\pi}\left(r_{\mathrm{v}}-1\right) \\
W_{\mu_{\mathrm{P}}}=-\int_{\mathrm{X}_{2}}^{\mathrm{X}_{1}} F_{\mu_{\mathrm{P}}}(x) \mathrm{d} x=\frac{2 v_{\max } \mu_{\mathrm{P}} X_{2}}{\pi}\left(r_{\mathrm{v}}-1\right)
\end{array}\right.
$$

For the two irreversible adiabatic processes, the compression and expansion efficiencies ${ }^{8,12,13}$

$$
\eta_{\mathrm{c}}=\frac{W_{1 \rightarrow 2 \mathrm{~S}}}{W_{1 \rightarrow 2}}=\frac{T_{2 \mathrm{~S}}-T_{1}}{T_{2}-T_{1}}
$$

and

$$
\eta_{\mathrm{e}}=\frac{W_{3 \rightarrow 4}}{W_{3 \rightarrow 4 \mathrm{~S}}}=\frac{T_{3}-T_{4}}{T_{3}-T_{4 \mathrm{~S}}}
$$

can be used to describe the irreversibility of these processes. When the friction works in adiabatic processes are taken into account, the compression and expansion efficiencies may be rewritten as

$$
\begin{aligned}
\eta_{\mathrm{c}} & =\frac{W_{1 \rightarrow 2 \mathrm{~S}}}{W_{1 \rightarrow 2}}=\frac{W_{1 \rightarrow 2 \mathrm{~S}}}{W_{1 \rightarrow 2 \mathrm{~S}}+W_{\mu_{\mathrm{C}}}} \\
& =\frac{C_{\mathrm{V}}\left(T_{2 \mathrm{~S}}-T_{1}\right)}{C_{\mathrm{V}}\left(T_{2 \mathrm{~S}}-T_{1}\right)+W_{\mu_{\mathrm{C}}}}
\end{aligned}
$$

and

$$
\begin{aligned}
\eta_{\mathrm{e}} & =\frac{W_{3 \rightarrow 4}}{W_{3 \rightarrow 4 \mathrm{~S}}}=\frac{W_{3 \rightarrow 4 \mathrm{~S}}-W_{\mu_{\mathrm{P}}}}{W_{3 \rightarrow 4 \mathrm{~S}}} \\
& =\frac{C_{\mathrm{V}}\left(T_{3}-T_{4 \mathrm{~S}}\right)-W_{\mu_{\mathrm{P}}}}{C_{\mathrm{V}}\left(T_{3}-T_{4 \mathrm{~S}}\right)}
\end{aligned}
$$

Using equations (5)-(8) and the adiabatic equations of an ideal gas

$$
\frac{T_{2 \mathrm{~S}}}{T_{1}}=\frac{T_{3}}{T_{4 \mathrm{~S}}}=\left(\frac{V_{1}}{V_{2}}\right)^{\gamma-1}=r_{\mathrm{v}}^{\gamma-1}
$$

one can obtain

$$
\begin{aligned}
T_{2} & =T_{1}+\left(T_{2 \mathrm{~S}}-T_{1}\right) / \eta_{\mathrm{c}} \\
& =T_{1} r_{\mathrm{v}}^{\gamma-1}+\frac{2 v_{\max } \mu_{\mathrm{C}} X_{2}}{\pi C_{\mathrm{V}}}\left(r_{\mathrm{v}}-1\right) \\
T_{4} & =T_{3}+\eta_{\mathrm{e}}\left(T_{4 \mathrm{~S}}-T_{3}\right) \\
& =T_{3} r_{\mathrm{v}}^{1-\gamma}+\frac{2 v_{\max } \mu_{\mathrm{P}} X_{2}}{\pi C_{\mathrm{V}}}\left(r_{\mathrm{v}}-1\right) \\
\eta_{\mathrm{c}} & =\frac{r_{\mathrm{v}}^{\gamma-1}-1}{r_{\mathrm{v}}^{\gamma-1}-1+2 v_{\max } \mu_{\mathrm{C}} X_{2}\left(r_{\mathrm{v}}-1\right) /\left(\pi C_{\mathrm{V}} T_{1}\right)}
\end{aligned}
$$

and

$$
\eta_{\mathrm{e}}=\frac{1-r_{\mathrm{v}}^{1-\gamma}-2 v_{\max } \mu_{\mathrm{P}} X_{2}\left(r_{\mathrm{v}}-1\right) /\left(\pi C_{\mathrm{V}} T_{3}\right)}{1-r_{\mathrm{v}}^{1-\gamma}}
$$

where $\gamma=C_{\mathrm{P}} / C_{\mathrm{V}}$ is the specific heat ratio. It is clearly seen from equations (12) and (13) that the compression and expansion efficiencies depend closely on the maximum piston velocity $v_{\max }$, friction coefficients $\mu_{\mathrm{C}}$ and $\mu_{\mathrm{P}}$, the specific heat ratio $\gamma$ and other parameters. It shows that the cycle model established here is more general than that adopted in Refs. 8, 12 and 13, in which the compression and expansion efficiencies of the irreversible adiabatic processes are only considered to be two given parameters.

For a real Otto engine, heat transfer irreversibility between the working substance and cylinder wall is not negligible. ${ }^{14}$ Heat added to the working substance by combustion may be given by the linear relation $6,7,9,14$

$$
Q_{23}=\alpha-\beta\left(T_{2}+T_{3}\right)
$$

where $\alpha$ and $\beta$ are two constants related to combustion and heat transfer. It is often assumed that heat leak losses through the cylinder wall are proportional to the average temperature of both the working substance and cylinder wall ${ }^{15}$ and that the wall temperature is a constant. Furthermore, it is often assumed that heat transfer between the working substance and cylinder wall obeys a Newtonian law, ${ }^{15-21}$ so that equation (14) may be rewritten as

$$
\begin{aligned}
Q_{23} & =Q_{\mathrm{T}}-K\left[\left(T_{2}+T_{3}\right) / 2-T_{0}\right] \\
& =Q_{\mathrm{T}}-\beta\left(T_{2}+T_{3}-2 T_{0}\right)
\end{aligned}
$$

where $K=2 \beta$ is the thermal conductance between working substance and cylinder wall, $T_{0}$ is the average temperature of cylinder wall, and $Q_{\mathrm{T}}$ is the total heat released by combustion.

\section{Work output and efficiency}

Using equations (4), (10), (11) and (15), one can derive the expressions of work output and efficiency of the Otto heat engine as

$$
\begin{aligned}
W & =Q_{23}-Q_{41}=C_{\mathrm{V}}\left(T_{3}-T_{2}\right)-C_{\mathrm{V}}\left(T_{4}-T_{1}\right) \\
& =C_{\mathrm{V}} T_{1}\left[\left(1+T_{3} / T_{1}+a\right)-\left(T_{3} / T_{1}\right) r_{\mathrm{v}}^{1-\gamma}-r_{\mathrm{v}}^{\gamma-1}-a r_{\mathrm{v}}\right] \\
& =C_{\mathrm{V}} T_{1} W^{*}
\end{aligned}
$$




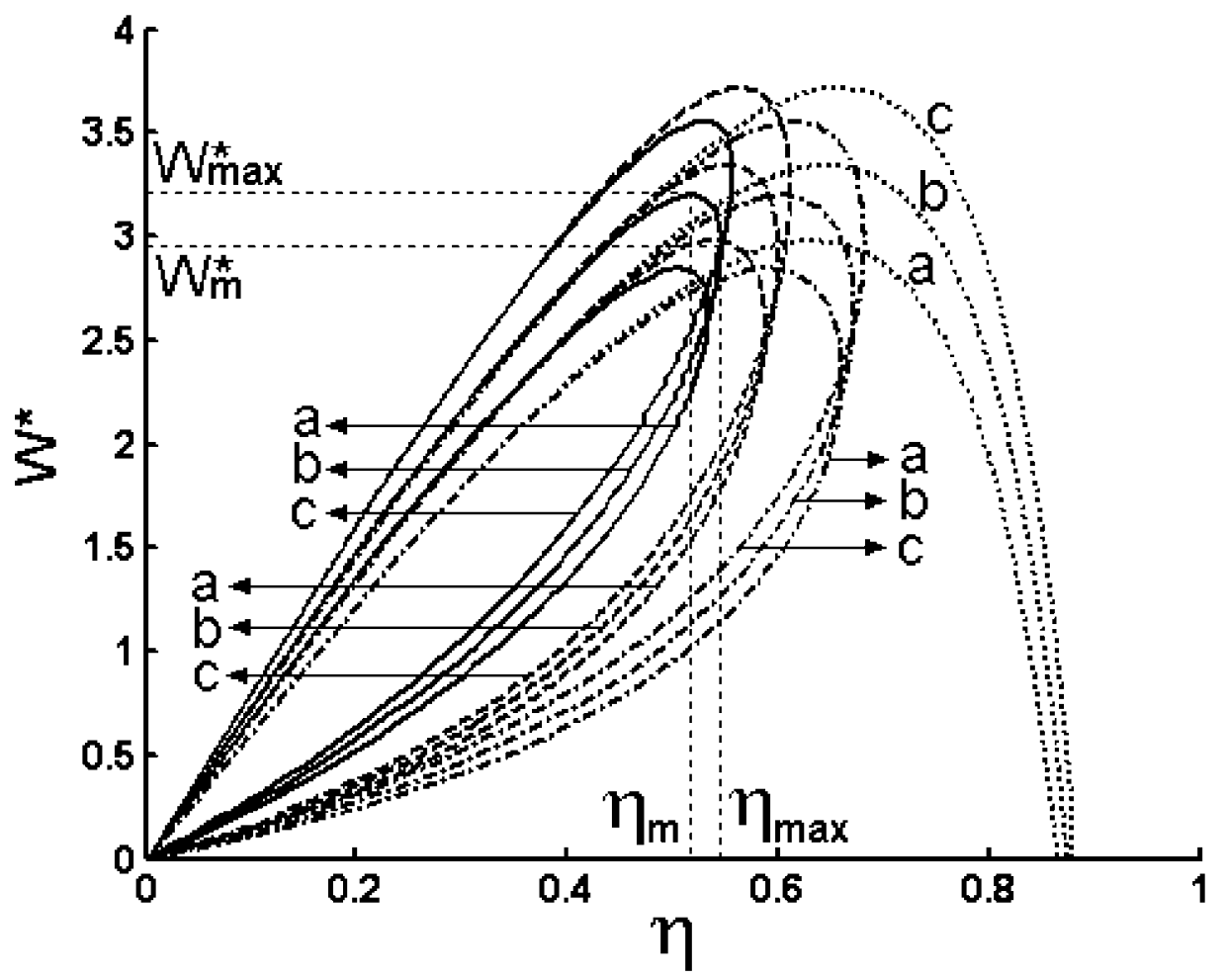

$2 W^{*}-\eta$ curves of irreversible Otto heat engine for parameters $T_{1}=350 \mathrm{~K}, T_{0}=T_{1}, \gamma=1.4, C_{\mathrm{V}}=0.7165 \mathrm{~J} \mathrm{~K}^{-1}, X_{2}=0.01 \mathrm{~m}$ and $v_{\max }=13.2 \mathrm{~m} \mathrm{~s}^{-1}$. The solid, dashed, dash dot and dotted curves correspond to the cases of $\beta / C_{\mathrm{V}}=0.1$, $\mu_{\mathrm{C}}=12.9 \mathrm{~kg} \mathrm{~s}^{-1} ; \beta / C_{\mathrm{V}}=0.1, \mu_{\mathrm{C}}=0 ; \beta / C_{\mathrm{V}}=0, \mu_{\mathrm{C}}=12.9 \mathrm{~kg} \mathrm{~s}^{-1}$; and $\beta / C_{\mathrm{V}}=\mu_{\mathrm{C}}=0$ respectively. Curves a, b and c correspond to the cases of $T_{3}=2600,2800$ and $3000 \mathrm{~K}$ respectively.

and

$$
\begin{aligned}
& \eta=\frac{W}{Q_{\mathrm{T}}}=\frac{C_{\mathrm{V}}\left(T_{3}-T_{2}\right)-C_{\mathrm{V}}\left(T_{4}-T_{1}\right)}{Q_{23}+\beta\left(T_{2}+T_{3}-2 T_{0}\right)} \\
& =\frac{\left(1+T_{3} / T_{1}+a\right)-\left(T_{3} / T_{1}\right) r_{\mathrm{v}}^{1-\gamma}-r_{\mathrm{v}}^{\gamma-1}-a r_{\mathrm{v}}}{\left(\beta / C_{\mathrm{V}}+1\right)\left(T_{3} / T_{1}\right)-2\left(\beta / C_{\mathrm{V}}\right)\left(T_{0} / T_{1}\right)+\left(\beta / C_{\mathrm{V}}-1\right)\left[b\left(r_{\mathrm{v}}-1\right)+r_{\mathrm{v}}^{\gamma-1}\right]}
\end{aligned}
$$

where $a=\frac{2 v_{\max } X_{2}\left(\mu_{\mathrm{C}}+\mu_{\mathrm{P}}\right)}{\pi C_{\mathrm{V}} T_{1}}$ and $b=\frac{2 v_{\max } X_{2} \mu_{\mathrm{C}}}{\pi C_{\mathrm{V}} T_{1}}$ are dimensionless parameters, and $W^{*}=W /\left(C_{\mathrm{V}} T_{1}\right)=\left(1+T_{3} / T_{1}+a\right)-$ $\left(T_{3} / T_{1}\right) r_{\mathrm{v}}^{1-\gamma}-r_{\mathrm{v}}^{\gamma-1}-a r_{\mathrm{v}}$ is the dimensionless work output. It is seen from equation (17) that the efficiency of the cycle is closely dependent on heat leak losses which must be considered in practice.

\section{Optimal performance characteristics}

It is seen from equations (16) and (17) that the work output and efficiency of the cycle depend not only on a set of mechanical parameters, but also on a set of thermodynamic parameters. Using equations (16) and (17) and the engine parameters reported by Mozurkewich and Berry, ${ }^{1}$ the authors can plot the characteristic curves of dimensionless work output versus efficiency, as shown in Fig. 2, where parameters $T_{1}=350 \mathrm{~K}, \quad T_{0}=T_{1}, \quad \gamma=1.4, \quad C_{\mathrm{V}}=0.7165 \mathrm{~J} \mathrm{~K}^{-1}, \quad X_{2}=$ $0.01 \mathrm{~m}, v_{\max }=13.2 \mathrm{~m} \mathrm{~s}^{-1}, \mu_{\mathrm{C}}=12.9 \mathrm{~kg} \mathrm{~s}^{-1}$ and $\beta / C_{\mathrm{V}}=$ $0 \cdot 1$ are chosen. $W_{\max }^{*}$ and $\eta_{\max }$ represent the maximum dimensionless work output and maximum efficiency, while $\eta_{\mathrm{m}}$ and $W_{\mathrm{m}}^{*}$ represent efficiency at the maximum work output and dimensionless work output at the maximum efficiency respectively.

Curves in Fig. 2 show clearly that for an irreversible Otto heat engine, there exist a maximum work output and a maximum efficiency. Using equations (16) and (17) and their extremal conditions, the authors can prove that when the work output and efficiency attain thei $\mathrm{r}$ maxima, the corresponding compression ratios $r_{\mathrm{vW}}$ and $r_{\mathrm{v} \eta}$ are determined by

$$
[a /(\gamma-1)] r_{\mathrm{vW}}+r_{\mathrm{vW}}^{\gamma-1}-\left(T_{3} / T_{1}\right) r_{\mathrm{vW}}^{1-\gamma}=0
$$

and

$$
m_{1} r_{\mathrm{v} \eta}^{\gamma}+m_{2} r_{\mathrm{v} \eta}+m_{3} r_{\mathrm{v} \eta}^{2-\gamma}+m_{4} r_{\mathrm{v} \eta}^{\gamma-1}+m_{5} r_{\mathrm{v} \eta}^{1-\gamma}+m_{6}=0(19)
$$

respectively, where

$$
\begin{aligned}
& m_{1}=\left(\beta / C_{\mathrm{V}}-1\right)(a-b)(2-\gamma) \\
& m_{2}=a m_{7}+b\left(\beta / C_{\mathrm{V}}-1\right)\left(1+T_{3} / T_{1}\right) \\
& m_{3}=-b \gamma\left(\beta / C_{\mathrm{V}}-1\right)\left(T_{3} / T_{1}\right) \\
& m_{4}=(\gamma-1)\left[\left(\beta / C_{\mathrm{V}}-1\right)\left(1+T_{3} / T_{1}+a-b\right)+m_{7}\right] \\
& m_{5}=(\gamma-1)\left(T_{3} / T_{1}\right)\left[b\left(\beta / C_{\mathrm{V}}-1\right)-m_{7}\right] \\
& m_{6}=2\left(\beta / C_{\mathrm{V}}-1\right)\left(T_{3} / T_{1}\right)(1-\gamma)
\end{aligned}
$$

and

$$
m_{7}=\left(\beta / C_{\mathrm{V}}+1\right)\left(T_{3} / T_{1}\right)-2\left(\beta / C_{\mathrm{V}}\right)\left(T_{0} / T_{1}\right)
$$

Figure 2 also shows that when the Otto heat engine is operated in those parts of the $W-\eta$ curve which has a positive slope, work output will decrease as efficiency is decreased. These regions are not the optimally operating regions of heat engine. Obviously, the optimally operating region should be situated in the part of $W-\eta$ curve which has a negative slope. Thus, the optimal ranges of work output and efficiency should be 


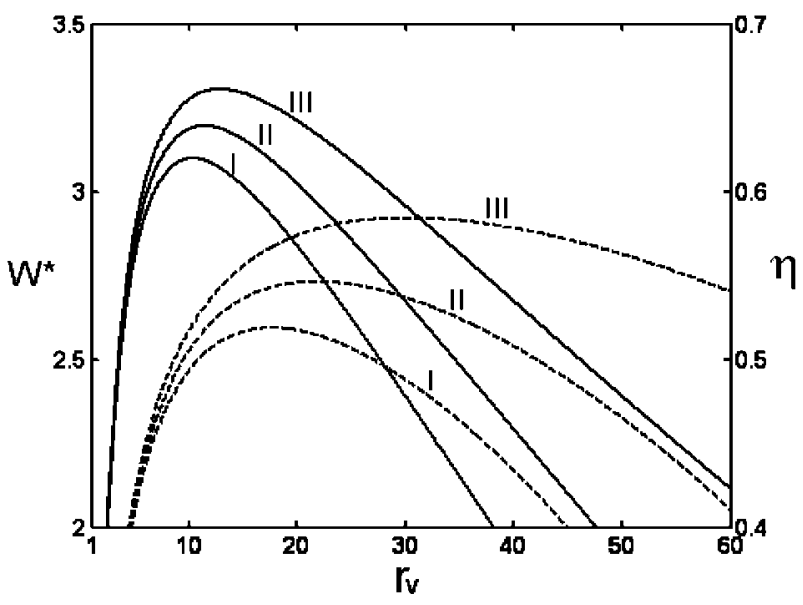

$3 W^{*}-r_{\mathrm{v}}$ (solid lines) and $\eta-r_{\mathrm{v}}$ (dashed lines) curves of irreversible Otto heat engine for parameters $T_{3}=2800 \mathrm{~K}$ and $\mu_{\mathrm{C}}=12.9 \mathrm{~kg} \mathrm{~s}^{-1}$ : values of other parameters are same as those used in Fig. 2; curves I, II and III correspond to cases of $v_{\max }=23.2,13.2$ and $3.2 \mathrm{~m} \mathrm{~s}^{-1}$ respectively

$W_{\mathrm{m}} \leqslant W \leqslant W_{\max }$

and

$$
\eta_{\mathrm{m}} \leqslant \eta \leqslant \eta_{\max }
$$

According to equations (21) and (22), the authors can determine the optimal region of compression ratio $r_{\mathrm{v}}$ for an irreversible Otto heat engine as

$$
r_{\mathrm{vW}} \leqslant r_{\mathrm{v}} \leqslant r_{\mathrm{v} \eta}
$$

This conclusion can also be explained by Fig. 2. In the region of $r_{\mathrm{v}}<r_{\mathrm{vW}}$, both work output and efficiency will decrease as compression ratio is decreased, while in the region of $r_{\mathrm{v}}>r_{\mathrm{v} \eta}$, both work output and efficiency will decrease as compression ratio is increased. Obviously, the regions of $r_{\mathrm{v}}<r_{\mathrm{vW}}$ and $r_{\mathrm{v}}>r_{\mathrm{v} \eta}$ are not optimal although the heat engine can work in these regions. Thus, $r_{\mathrm{v} \eta}$ and $r_{\mathrm{vW}}$ are also two important parameters, which determine the upper and lower bounds of optimised compression ratio respectively. In the design of Otto heat engine, engineers should choose a reasonable compression ratio according to equations (18), (19) and (23), so that the engine can be operated in optimal region.

According to equations (10), (11) and (23), the authors can further determine the optimal regions of temperatures $T_{2}$ and $T_{4}$ as

$$
T_{2 \mathrm{~W}} \leqslant T_{2} \leqslant T_{2 \eta}
$$

and

$$
T_{4 \mathrm{~W}} \geqslant T_{4} \geqslant T_{4 \eta}
$$

respectively, where $T_{2 \mathrm{~W}}=T_{1} r_{v \mathrm{~W}}^{\gamma-1}+\frac{2 v_{\max } \mu_{\mathrm{C}} X_{2}}{\pi C_{\mathrm{V}}}\left(r_{\mathrm{vW}}-1\right)$,

$T_{2 \eta}=T_{1} r_{\mathrm{v} \eta}^{\gamma-1}+\frac{2 v_{\max } \mu_{\mathrm{C}} X_{2}}{\pi C_{\mathrm{V}}}\left(r_{\mathrm{v} \eta}-1\right)$,

$T_{4 \mathrm{~W}}=T_{3} r_{\mathrm{vW}}^{1-\gamma}+\frac{2 v_{\max } \mu_{\mathrm{P}} X_{2}}{\pi C_{\mathrm{V}}}\left(r_{\mathrm{vW}}-1\right)$,

$T_{4 \eta}=T_{3} r_{\mathrm{v} \eta}^{1-\gamma}+\frac{2 v_{\max } \mu_{\mathrm{P}} X_{2}}{\pi C_{\mathrm{V}}}\left(r_{\mathrm{v} \eta}-1\right)$.

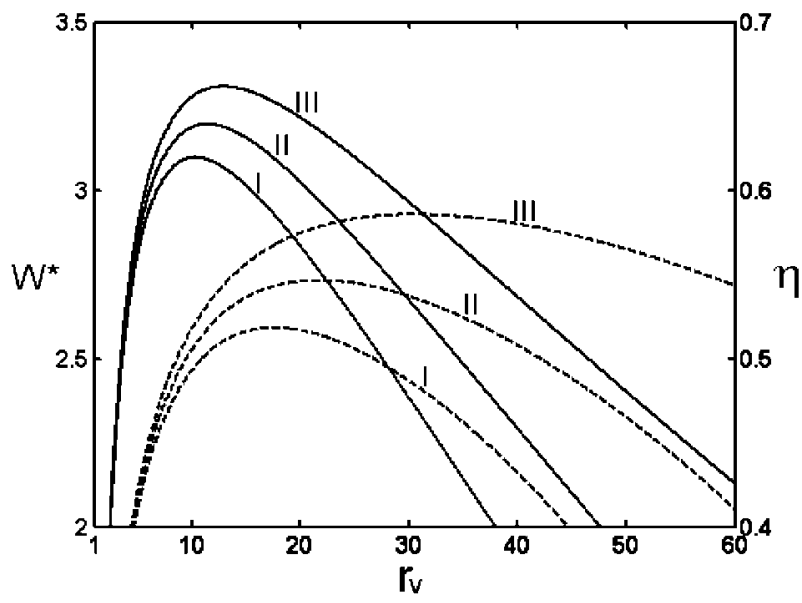

$4 W^{*}-r_{\mathrm{v}}$ (solid lines) and $\eta-r_{\mathrm{v}}$ (dashed lines) curves of irreversible Otto heat engine for parameters $T_{3}=2800 \mathrm{~K}$ and $v_{\max }=13.2 \mathrm{~m} \mathrm{~s}^{-1}$ : values of other parameters are same as those used in Fig. 2; curves I, II and III correspond to cases of $\mu_{\mathrm{C}}=22.9,12.9$ and $2.9 \mathrm{~kg} \mathrm{~s}^{-1}$ respectively

\section{Discussion}

\section{Influence of highest temperature $T_{3}$}

It is clearly seen from Fig. 2 that the work output and efficiency increase quickly as temperature $T_{3}$ increases, which means that the higher $T_{3}$, the better the performance of heat engine. However, $T_{3}$ is always limited by the melting points of engine materials and other physical properties. ${ }^{11}$ Furthermore, Fig. 2 shows that the optimal ranges of work output and efficiency also increase with increasing $T_{3}$, while both $r_{\mathrm{vW}}$ and $r_{\mathrm{v} \eta}$ increase slightly with increasing $T_{3}$. This characteristic will be advantageous to the design and manufacture of the Otto heat engine. Moreover, the numerical calculations indicate that the higher $T_{3}$, the larger the optimal regions of $T_{2}$ and $T_{4}$, because $T_{2 \eta}$ and $T_{4 \mathrm{~W}}$ increase more quickly than $T_{4 \eta}$ and $T_{2 \mathrm{~W}}$.

\section{Influence of friction force}

As shown in Figs. 3 and 4, the work output and efficiency decrease quickly as the maximum piston velocity $v_{\max }$ and/or the friction coefficients $\mu_{\mathrm{C}}$ and $\mu_{\mathrm{P}}$ increase. It is natural, because the friction force is proportional to the maximum piston velocity and friction coefficients. It is also seen from Figs. 3 and 4 that $r_{\mathrm{v} \eta}$ will increase more quickly than $r_{\mathrm{vW}}$ as the maximum piston velocity or the friction coefficient is decreased, so that the optimal region of the compression ratio increases. Through numerical calculations it can be further proved that $T_{2 \mathrm{~W}}$ and $T_{2 \eta}$ increase while $T_{4 \eta}$ and $T_{4 \mathrm{~W}}$ decrease as the maximum piston velocity or the friction coefficient is decreased.

When the friction force in the adiabatic processes is negligible, i.e. $\mu_{\mathrm{C}}=\mu_{\mathrm{P}} \rightarrow 0$ or $v_{\max } \rightarrow 0$, equations (16) and (17) can be simplified as respectively ${ }^{9}$

$$
W=C_{\mathrm{V}} T_{1}\left[1+T_{3} / T_{1}-\left(T_{3} / T_{1}\right) r_{\mathrm{v}}^{1-\gamma}-r_{\mathrm{v}}^{\gamma-1}\right]
$$

and

$$
\eta=
$$


In such a case, the performance characteristic curves of the Otto heat engine are shown by the dashed curves in Fig. 2.

\section{Influence of heat leak losses}

It is seen from equations (16) and (17) that heat leak losses always reduce the efficiency of Otto heat engine although the work output is independent of them, as shown clearly in Fig. 2. Moreover, from numerical calculations the authors also find that $r_{\mathrm{v} \eta}$ will increase when the heat leak losses are decreased, so that the optimal region of the compression ratio increases because $r_{\mathrm{vW}}$ is not affected by heat leak losses.

When the heat leak losses are negligible, i.e, $\beta / C_{\mathrm{V}} \rightarrow 0$, the expression of work output is still given by equation (16), while the efficiency may be simplified as

$$
\eta=\frac{\left(1+T_{3} / T_{1}+a\right)-\left(T_{3} / T_{1}\right) r_{\mathrm{v}}^{1-\gamma}-r_{\mathrm{v}}^{\gamma-1}-a r_{\mathrm{v}}}{T_{3} / T_{1}+b-r_{\mathrm{v}}^{\gamma-1}-b r_{\mathrm{v}}}
$$

In such a case, the performance characteristic curves of Otto heat engine are shown by the dash dot curves in Fig. 2.

\section{Reversible cycle}

When the losses due to both friction force and heat leak are negligible, i.e. $\mu_{\mathrm{C}}=\mu_{\mathrm{P}} \rightarrow 0$ and $\beta / C_{\mathrm{V}} \rightarrow 0$, the expression of work output is still given by equation (26), while the efficiency may be further simplified as ${ }^{2,8,9}$

$$
\eta=1-r_{\mathrm{v}}^{1-\gamma}
$$

In such a case, the characteristic curves of Otto heat engine are shown by the dotted curves in Fig. 2. Obviously, the efficiency is only a monotonically increasing function of the compression ratio and independent of temperature. The dimensionless work output versus efficiency curves become parabolic, which are just the results of a reversible Otto heat engine.

\section{Conclusions}

The present cycle model avoids not only the usual hypotheses of endoreversible heat engines, ${ }^{5,11,17}$ but also the simple description of irreversible heat engines with given compression and expansion efficiencies ${ }^{8,12,13}$ or the piston mean velocity. ${ }^{2-4}$ Work output and efficiency of the cycle are maximised and optimum criteria of some important parameters are given. It is found that the increase in heat leak losses always reduces the efficiency and the friction losses always reduce both the work output and efficiency. The highest temperature $T_{3}$ of working substance and maximum motion velocity $v_{\max }$ of the piston have great influence on the work output and efficiency. The results obtained here are expected to provide a significant guidance for the performance improvement and optimal design of practical Otto heat engines.

\section{References}

1. M. Mozurkewich and R. S. Berry: J. Appl. Phys., 1982, 53, 34-42.

2. F. Angulo-Brown, J. Fernández-Betanzos and C. A. Diaz-Pico: Eur. J. Phys., 1994, 15, 38-42.

3. F. Angulo-Brown, J. A. Rocha-Martinez and T. D. NavarreteGonzalez: J. Phys. D, Appl. Phys., 1996, 29, 80-83.

4. A. C. Hernández, J. M. M. Roco, A. Medina and S. Velasco: Eur. J. Phys., 1996, 17, 11-18.

5. C. Wu and D. A. Blank: Energy Convers. Mgmt., 1993, 34, 12551259.

6. L. Chen, C. Wu, F. Sun and S. Cao: Energy Convers. Mgmt., 1998, 39, 643-648.

7. S. A. Klein: J. Eng. Gas Turbines \& Power, 1991, 113, 511-513.

8. G. Aragón-González, A. Canales-Palma and A. León-Galicia: J. Phys. D, Appl. Phys., 2000, 33, 1403-1409.

9. J. Chen, Y. Zhao and J. He: Appl. Energ., 2006, 83, 228-238.

10. A. C. Hernández, A. Medina, J. M. M. Roco and S. Velasco: Eur. J. Phys., 1995, 16, 73-75.

11. H. S. Leff: Am. J. Phys., 1987, 55, 602-610.

12. L. Chen, J. Zheng, F. Sun and C. Wu: J. Phys. D, Appl. Phys., 2001, 34, 1727-1739.

13. G. Aragón-González, A. Canales-Palma, A. León-Galicia and M. Musharrafie-Martínez: J. Phys. D, Appl. Phys., 2003, 36, 280-287.

14. O. A. Ozsoysal: Energy Convers. Mgmt., 2006, 47, 1051-1062.

15. K. H. Hoffman, S. J. Watowich and R. S. Berry: J. Appl. Phys., 1985, 58, 2125-2134.

16. A. de Vos: Energy Convers. Mgmt., 1995, 36, 1-5.

17. L. Guzmán-Vargas, I. Reyes-Ramírez and N. Sánchez: J. Phys. D, Appl. Phys., 2005, 38, 1282-1291.

18. B. Andresen, M. H. Rubin and R. S. Berry: J. Phys. Chem., 1983, 87, 2704-2713.

19. A. de Vos, P. T. Landsberg, P. Baruch and J. E. Parrott: J. Appl. Phys., 1993, 74, 3631-3637.

20. F. Angulo-Brown, L. A. Arias-Hernández and R. Páez-Hernández: J. Phys. D, Appl. Phys., 1999, 32, 1415-1420.

21. A. de Vos: Energy Convers. Mgmt., 1997, 38, 311-317. 